\title{
New Simulation Methodology of 3D Surface Roughness Loss for Interconnects Modeling
}

\author{
Quan Chen and Ngai Wong \\ Department of Electrical and Electronic Engineering \\ The University of Hong Kong \\ quanchen@eee.hku.hk,nwong@eee.hku.hk
}

\begin{abstract}
Abstract-As clock frequencies exceed giga-Hertz, the extra power loss due to conductor surface roughness in interconnects and packagings is more evident and thus demands a proper accounting for accurate prediction of signal integrity and energy consumption. Existing techniques based on analytical approximation often suffer from a narrow valid range, i.e., small or large limit of roughness. In this paper, we propose a new simulation methodology for surface roughness loss that is applicable to general surface roughness and a wide frequency range. The method is based on $3 D$ statistical modeling of surface roughness and the numerical solution of scalar wave modeling (SWM) with the method of moments (MOM). The spectral stochastic collocation method (SSCM) is applied in association of random surface modeling to avoid the time-consuming Monte-Carlo (MC) simulation. Comparisons with existing methods in their respective valid region then verify the effectiveness of our approach.
\end{abstract}

\section{INTRODUCTION}

Surface roughness in metal conductor of interconnects and microelectronic packaging is sometimes due to process variations in fabrication and, more frequently, artificial roughening processes, e.g., electronic deposition and chemical etching, to enhance interfacial adhesion between dielectrics and conducting material. Fig. 1 visualizes the micro-topologies of two different surface roughness in PCB interconnects. It has been reported by measurements that the surface profile variation in roughened copper foil can cause an increase of resistivity by a factor of 2 to 3 in microwave frequencies [1]. Recent experimental studies can be found in [2], [3]. Such impact on resistivity breaks the predictive capacity of classical high-frequency relationship $R_{f} \propto \sqrt{f}$ for the frequencydependent resistance $R_{f}$. With the ever-increasing signaling rate, especially in off-chip communications of multi-processor architectures, an accurate estimation of rough surface effect is imperatively demanded for improved prediction of factors like insertion loss, signal integrity and thermomechanical reliability in interconnect-aware design methodologies.

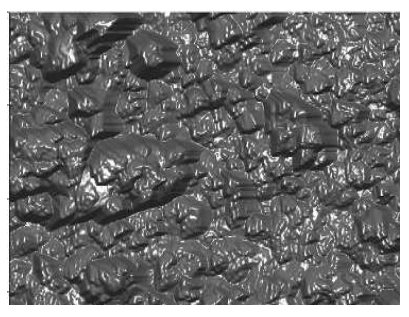

(a)

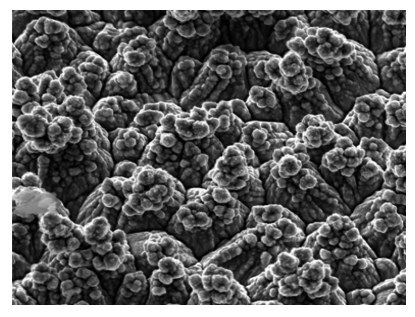

(b)
Fig. 1. Micro-graphs of surface roughness in PCB interconnects (a [4], b [5].

Work to investigate the rough surface loss has been done mainly within the context of electromagnetism. The earliest research can be dated back to 1940's when Morgan first studied the increased resistive power loss for a periodic $2 \mathrm{D}$ ridged structure [6]. The results were then fitted as an empirical formula (1), which has since then been the guideline model for quantifying surface-roughness-induced power loss in practice [7].

$$
\frac{P_{r}}{P_{s}}=\left[1+\frac{2}{\pi} \tan ^{-1}\left(1.4\left(\frac{\sigma}{\delta}\right)^{2}\right)\right] .
$$

Here $P_{r}$ and $P_{s}$ are the power loss of a rough surface conductor and its smooth surface counterpart, respectively. $\sigma$ is the standard deviation of surface height, $\delta=\sqrt{\rho /(\pi f \mu)}$ the skin depth, $\rho$ the conductor resistivity and $\mu$ the freespace permeability. As only $\sigma$ of the surface characteristics is included, (1) is subjected to frequent modifications to match field-measurement data from different environments where surface roughness depends on more than one parameter. Ref. [8] has shown that the power absorption enhancement factor of a 3D random rough surface can be much higher than that of a 2D one and, based on random surface modeling, derived a closed-form formula for this factor using secondorder small perturbation method (SPM2). Nevertheless, SPM2 
requires the roughness to be mild enough to validate the preassumption of "perturbation", which limits its application to a narrow range of cases with small roughness or at low frequencies. Another method is the hemispherical boss modeling (HBM) [5], which has a long history but regains attention only recently. It is distinguished by modeling surface irregularities as size-controlled hemispherical bosses distributed (regularly or randomly) on a flat plane. By exploiting the analytical solution of electromagnetic wave scattering from a conducting sphere the energy absorbed by the protrusion can be readily obtained. HBM is a gross assumption since surface roughness in real interconnects is quite diverse and is therefore difficult to be modeled by simple bosses. In addition, HBM primarily works under scenarios, as the opposite of SPM2, with large scale roughness or at very high frequencies, where the skin depth is small compared to radii of the bosses. Consequently, there is still a lack of efficient simulation method for evaluating surface roughness loss at intermediate range, where the skin depth is at the same order of the dimension of roughness, which is often the case in practice.

In this paper we propose a robust methodology to simulate the impact of surface roughness with diverse roughness patterns in a wide frequency range. A rough surface is modeled by a parameterized stochastic processes. The new formulation transforms the complicated electromagnetic (EM) problem into a simpler scalar form by regarding the EM wave as a scalar energy flux. In this way the extra loss caused by surface roughness can be approximated by the energy flux absorbed by the rough surface. The scalar wave modeling (SWM) therefore established are solved by the method of moments (MOM), which is more robust to irregular surface topologies. An efficient stochastic solver, spectral stochastic collocation method (SSCM) [9], is applied to compute the mean value of loss enhancement factor. Numerical comparisons with existing techniques in their respective valid regions are then conducted to verify the effectiveness of the proposed methodology.

\section{Characterization OF 3D RANDOM ROUGH SURFACE}

For a 3D random rough surface of conductor, we describe the surface height $f\left(\bar{r}_{\perp}\right)$ as a stationary stochastic process, where $\bar{r}_{\perp}=(x, y)$ falls on the mean plane. A stochastic process is generally characterized by the probability density function (PDF) and the spatial correlation function (CF) $C\left(\bar{r}_{\perp i}, \bar{r}_{\perp j}\right)$. Without loss of generality, we use the common Gaussian PDF (2) in this paper associated with different CFs.

$$
\operatorname{Pdf}\left(f\left(\bar{r}_{\perp}\right)\right)=\frac{1}{\sqrt{2 \pi} \sigma} \exp \left(-\frac{f^{2}\left(\bar{r}_{\perp}\right)}{2 \sigma^{2}}\right),
$$

where $\sigma$ is the standard deviation and the mean plane is on $f=0$. An important feature of this characterization is that the parameters of stochastic process, e.g., $\sigma$ and $C$, can be quantitatively extracted from real interconnect surface by measuring surface height as a function of

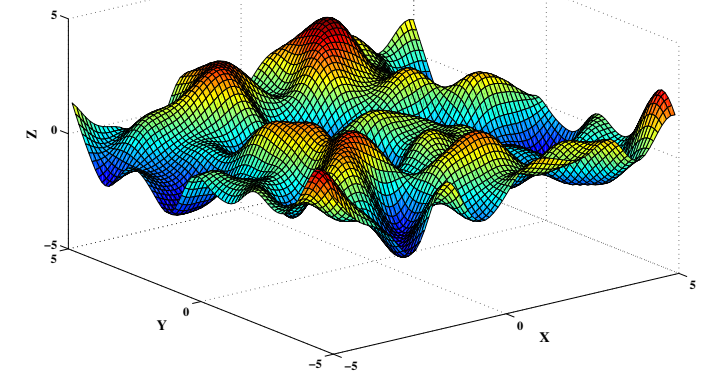

Fig. 2. Simulated 3D random rough surface with Gaussian $\mathrm{CF}$ and $\sigma=\eta=1 \mu m$.

position. Therefore, different surface roughness in reality can be reproduced and simulated by properly parameterizing the stochastic processes. Fig. 2 shows a simulated $3 \mathrm{D}$ random rough surface with the common Gaussian $\mathrm{CF}$ $\mathrm{C}(d)=\sigma^{2} \exp \left(-\frac{d^{2}}{\eta^{2}}\right)$, where $d=\left|\bar{r}_{\perp i}-\bar{r}_{\perp j}\right|$ and $\eta$ is the correlation length.

\section{ScAlar WAVE MODELING}

In principle, electromagnetic wave theory can be used to rigorously determine the field scattered or absorbed by a conducting body, which has been extensively studied for several decades [10]. Direct application of numerical EM simulation to predict surface roughness loss in microscopic interconnects, however, has the following two difficulties: 1) The wavelength in dielectrics (in the order of $\mathrm{cm}$ ) is large compared to the dimension of conductor surface (in the order of $\mu \mathrm{m}$ ). This corresponds to the well-known low-frequency problem in traditional EM scattering problem from metallic objects, in which the solution of integral equation will become unstable due to the dominant impact of hyper-singular integral operator [11]. Remedies of such problem include using highorder loop-star [12] or modifying the integral operators [13], all of which will significantly increase the complexity of calculation. 2) The computation is intensive as a rough surface may need a fine discretization to properly capture the details of surface variation. The situation will be even worse if the low-frequency problem (requires higher-order basis or operator) and random surface modeling (requires multiple sampling) are taken into account.

To balance the cost and performance, we choose the scalar wave theory instead of EM wave theory to formulate the energy loss due to the surface roughness in interconnects. The scalar wave modeling (SWM) has long been used as a simplified substitute for vectorial EM wave to predict wave behavior and observe important phenomena in scattering problems [14]. It has been shown that the results of scalar wave modeling are well correlated to that from EM wave modeling in many scenarios, especially when the detailed field distribution is not required [15]. We consider a two- 
medium problem, where the upper half-space of rough interface $S$ is filled by dielectrics (medium 1 ) with electrical parameters $\epsilon_{1}, \mu$ and wavenumber $k_{1}=\omega \sqrt{\mu \epsilon_{1}}, \omega=2 \pi f$, and the lower half by conducting material (medium 2) with $\rho, \mu$ and $k_{2}=(1+j) / \delta$. One considers a scalar plane wave normally impinging on the rough surface $\psi_{i n}(\bar{r})=$ $\exp \left(-j k_{0} z\right)$, where $\bar{r}=(x, y, z)$, then the wave functions in the two media $\psi_{i}, i=1,2$ satisfy the coupled surface integral equation:

$$
\begin{aligned}
& \iint_{S} \mathrm{~d} s^{\prime} \hat{n}^{\prime} \cdot\left[G_{1}\left(\bar{r}, \bar{r}^{\prime}\right) \nabla^{\prime} \psi_{1}\left(\bar{r}^{\prime}\right)-\nabla^{\prime} G_{1}\left(\bar{r}, \bar{r}^{\prime}\right) \psi_{1}\left(\bar{r}^{\prime}\right)\right] \\
& +\psi_{1}(\bar{r})=\psi_{i n}(\bar{r}), \quad \bar{r}, \bar{r}^{\prime} \in S \\
& \iint_{S} \mathrm{~d} s^{\prime} \hat{n}^{\prime} \cdot\left[G_{2}\left(\bar{r}, \bar{r}^{\prime}\right) \nabla^{\prime} \psi_{2}\left(\bar{r}^{\prime}\right)-\nabla^{\prime} G_{2}\left(\bar{r}, \bar{r}^{\prime}\right) \psi_{2}\left(\bar{r}^{\prime}\right)\right] \\
& -\psi_{2}(\bar{r})=0, \quad \bar{r}, \bar{r}^{\prime} \in S
\end{aligned}
$$

where $\hat{n}$ is the unit normal vector pointing outward and $G_{i}, i=1,2$ is the scalar Green's function in the $i$ th medium

$$
G_{i}\left(\bar{r}, \bar{r}^{\prime}\right)=\frac{\exp \left(j k_{i}\left|\bar{r}-\bar{r}^{\prime}\right|\right)}{4 \pi\left|\bar{r}-\bar{r}^{\prime}\right|}
$$

It should be noted that, unlike electric or magnetic field, the scalar field $\psi$ does not have a physical meaning and, intuitively, can be viewed as the velocity potential of a flux of energy (an analogous concept is the sound pressure for acoustic wave). The essence of our formulation is to calculate the amount of energy absorbed by a rough surface subjected to a given incident energy flux, which serves as an approximation of the real EM energy absorbed by the same surface.

\section{A. Continuous Boundary Condition for Scalar Wave}

Conventionally, the scalar wave equation (3) is solved by the eliminating boundary condition assuming $\psi$ or $\hat{n} \cdot \nabla \psi$ vanishes on the boundary since only exterior wave is of interest in scattering problem. However, calculation energy absorption in our case requires mainly the knowledge of the field transmitted into the conductor. To this end, we develop an approximated continuous boundary condition reflecting the continuity of real EM field across the rough boundary. As indicated in [5], [8] the EM field distribution near the surface of practical quasi-transverse electromagnetic (quasiTEM) interconnect structures is similar with that induced by an incident transverse-magnetic (TM) wave. Since the wavelength in dielectric is much larger than the scale of surface roughness, the magnetic field $\bar{H}$ very close to the surface can be approximated by its tangential component $\bar{H}_{t}$. If we associate $\psi$ with a physical meaning as the magnitude of $\bar{H}_{t}$, the follow relationship holds:

$$
\begin{aligned}
\bar{H} & \approx \bar{H}_{t}=\hat{t} \psi \\
& \bar{E}_{t} \approx-\frac{1}{j \omega \epsilon} \hat{n} \times(\nabla \times(\hat{t} \psi))=\frac{1}{j \omega \epsilon} \hat{n} \cdot \nabla \psi,
\end{aligned}
$$

where $\hat{t}$ is the unit tangential vector and the second equality in $\bar{E}_{t}$ formula is due to $\hat{n} \cdot \hat{t}=0$. Recalling the continuity of tangential fields across the boundary $\bar{H}_{t 1}=\bar{H}_{t 2}, \bar{E}_{t 1}=\bar{E}_{t 2}$, we have the continuity in terms of $\psi$ and $\hat{n} \cdot \nabla \psi$

$$
\psi_{1}(\bar{r})=\psi_{2}(\bar{r}), \quad \hat{n} \cdot \nabla \psi_{1}(\bar{r})=\beta \cdot \hat{n} \cdot \nabla \psi_{2}(\bar{r})
$$

where $\beta=\epsilon_{1} / \epsilon_{2}=-j \omega \epsilon_{1} \rho$.

\section{B. Doubly-Periodic Surface Assumption}

Equation (3) should be fundamentally solved on the whole resemble of realistic conductor surfaces, which, however, makes the solution procedure computationally prohibitive. Yet significant simplification is achievable via exploiting the particular properties of rough surface problem: 1) The impact of surface roughness is local, i.e., mutual coupling between surface roughness on separated conductors or different surfaces of one conductor is negligible. 2) The roughness is globally uniform such that a global factor of loss enhancement can be obtained from local analysis [4]. 3) The spatial correlation of roughness is small compared to the size of conductor surface, which means the surface contains, for random modeling, many peaks and valleys, or for HBM, a large number of bosses. This has been confirmed by physical measurements [5]. In light of the above observations, a doubly-periodic condition [15] is applied to restrict the investigation domain within a small patch of the whole surface by enforcing $f(x+p L, y+q L)=f(x, y)$, where $L$ is the periodical length and $p$ and $q$ are integers. Recalling the continuous relation (6), (3) becomes

$$
\begin{aligned}
& \psi(\bar{r})+\iint_{L^{2}} \mathrm{~d} r^{\prime}\left[-\frac{\partial G_{1}^{p q}\left(\bar{r}, \bar{r}^{\prime}\right)}{\partial \bar{n}^{\prime}} \psi\left(\bar{r}^{\prime}\right)+\gamma G_{1}^{p q}\left(\bar{r}, \bar{r}^{\prime}\right) u\left(\bar{r}^{\prime}\right)\right] \\
& =\psi_{\text {in }}(\bar{r}) \\
& \psi(\bar{r})+\iint_{L^{2}} \mathrm{~d} r^{\prime}\left[\frac{\partial G_{2}^{p q}\left(\bar{r}, \bar{r}^{\prime}\right)}{\partial \bar{n}^{\prime}} \psi\left(\bar{r}^{\prime}\right)-G_{2}^{p q}\left(\bar{r}, \bar{r}^{\prime}\right) u\left(\bar{r}^{\prime}\right)\right]=0
\end{aligned}
$$

where $L^{2}$ denotes the $L \times L$ patch, $\psi=\psi_{2}$ and $u=$ $\sqrt{1+f_{x}^{2}+f_{y}^{2}} \hat{n} \cdot \nabla \psi$ with $f_{x}=\partial f / \partial x$. The periodic Green's function and its normal derivative are

$$
\begin{gathered}
G_{i}^{p q}=\sum_{p=-\infty}^{\infty} \sum_{q=-\infty}^{\infty} \frac{\exp \left(j k_{i}\left|\bar{r}-\bar{r}^{\prime}-\hat{x} p L-\hat{y} q L\right|\right)}{4 \pi\left|\bar{r}-\bar{r}^{\prime}-\hat{x} p L-\hat{y} q L\right|} \\
\frac{\partial G_{i}^{p q}\left(\bar{r}, \bar{r}^{\prime}\right)}{\partial n^{\prime}}=\sqrt{1+f_{x}^{2}+f_{y}^{2}} \hat{n} \cdot \nabla^{\prime} G_{i}^{p q},
\end{gathered}
$$

The periodic Green's function in (8) can be efficiently computed by the Ewald method [16], which requires very few terms to converge.

Discretizing (7) with the method of moments (MOM) and combining the unknowns, we obtain the following matrix equation

$$
\left[\begin{array}{cc}
A_{1} & \beta B_{1} \\
A_{2} & B_{2}
\end{array}\right]\left[\begin{array}{c}
\Psi \\
U
\end{array}\right]=\left[\begin{array}{c}
\Psi_{i n} \\
0
\end{array}\right] .
$$

Equation (9) can be efficiently solved in $O(N \log (N))$ complexity, where $N$ is the number of discretized elements, with numerical solvers such as the FFT-based iterative method [17] and the UV-multilevel partitioning method [18]. After the scalar wave function $\Psi$ and $U$ are solved, the energy absorbed 
by the lower medium beneath the investigation patcl computed by

$$
P_{r}=\int_{L^{2}} \mathrm{~d} r \frac{1}{2} \operatorname{Re}\left\{\psi^{*}(\bar{r}) u(\bar{r})\right\} .
$$

The energy absorption for smooth surface case is

$$
P_{s}=L^{2} /(2 \delta) .
$$

\section{Comparison with Vectorial EM Modeling}

The rigorous surface integral formulation based on theory of rough surface scattering from penetrable obj can be found in a number of literature, e.g., [11], [ [19]. The surface is generally discretized by RWG triangular elements [20], each of which is associated with two vectorial unknowns, usually the electric and magnetic currents. The size of resultant matrix equation, if converted to corresponding scalar equations, is roughly $6 N \times 6 N$. If the low-frequency problem is taken into account, the common remedies, e.g, using high-order basis function or high-order operator, will significantly increase the complexity of calculation. In contrast, SWM results in a problem with the size of only $2 N \times 2 N$, which contributes a significant reduction in solution time and memory storage. Since no hyper-singular operator, i.e, second derivative of Green's function, appears in (3), the formulation is free of the low-frequency problem. The scalar nature of unknowns also allows smooth rectangular basis function to be applied to discretized the integral operator, rendering further computational saving compared to the RWG basis.

\section{Spectral Stochastic Collocation Method}

The equation (9) is solved only for a deterministic surface, i.e, the surface heights and surface derivatives must be explicitly given. If the surface roughness is modeled by a stochastic process described in Section II, (9) becomes a stochastic integral equation with the $N$ correlated random variables. The Monte-Carlo simulation is widely used to solve the stochastic integral equation by an extensive sampling of the solution space, but to guarantee convergence the number of samples might be prohibitively large, i.e., 5000 sampling points may be needed for convergence to within $1 \%$. To enhance the efficiency, we apply the recently-proposed method SSCM [9] to compute the statistical model of rough surface loss. The essence of SSCM is to model the solution of stochastic integral equation as the Homogeneous Chaos (HC) expansion in term of a set of independent random variables obtained from the original $N$ surface heights. Then the coefficients of $\mathrm{HC}$ expansion can be efficiently determined by the Sparse Grid (SG) quadrature. The advantage of SSCM is that it can achieve an second-order accuracy with much less sampling points than MC simulation. Details of SSCM can be referred to [9].

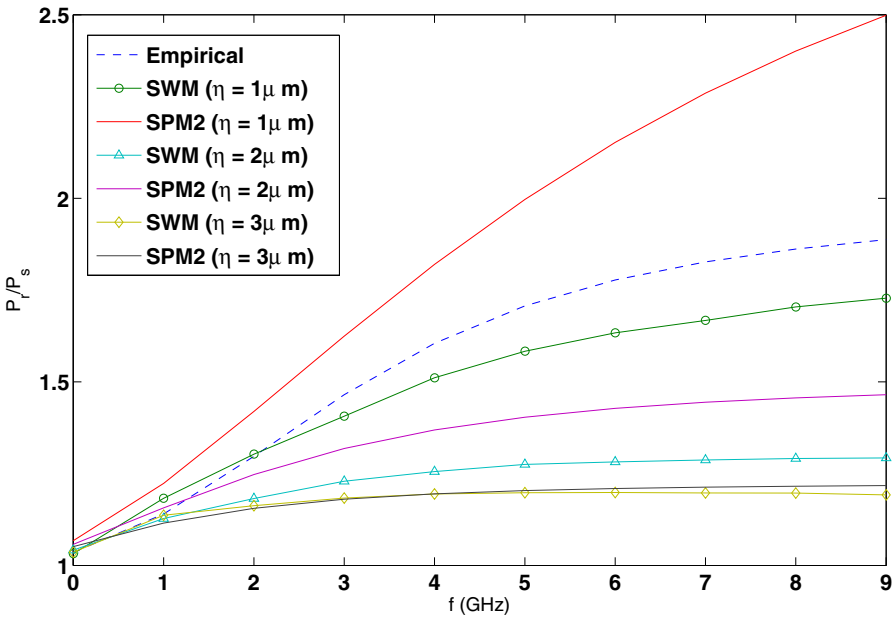

Fig. 3. SWM vs. SPM2 and empirical formula for Gaussian CF $(\sigma=1 \mu m, \eta=1,2,3 \mu m)$.

\section{NUMERICAL RESULTS}

In the following numerical experiments, we assume a conductor with the resistivity of $1.67 \mu \Omega \cdot \mathrm{cm}$ and a dielectric with the relative permittivity of common silicon dioxide (3.7). The periodic length $L$ of is $5 \eta$ and the discretized interval is $\eta / 8$. The surface roughness loss used for comparisons are their mean values computed by SSCM. All programming and simulation were done with Matlab on a desktop PC with $2.4 \mathrm{GHz}$ and $1 \mathrm{G}$ memory.

We first compare SWM with SPM2, which is known to be accurate for small roughness while invalid for large roughness. In Fig. 3 results of SWM and SPM2 are obtained for Gaussian surface with various levels of roughness, i.e., $\sigma$ is fixed at $1 \mu \mathrm{m}$ while $\eta$ varies from $1 \mu \mathrm{m}$ to $3 \mu \mathrm{m}$. For the most smooth case $(\eta=3 \mu m)$, the two methods are in a perfect match. The deviation, however, gradually grows as degree of roughness increases and becomes significant for the most rough case of $\eta=3 \mu \mathrm{m}$. We also plot the results from the empirical formula (1). As expected, (1) cannot differentiate these distinct cases as they have the same $\sigma$.

We further compare SWM with SPM2 using the CF (12) extracted from practical measurement data [4]. It has been shown in [4] that under this roughness setting SPM2 can give an accurate prediction of surface roughness loss. The testing frequency is from $100 \mathrm{MHz}$ to $10 \mathrm{GHz}$. As shown in Fig. 4, there is a good agreement between SWM and SPM2. The above two experiments suggest that SWM is accurate for small roughness cases. It should be noted that HBM is not applicable for these scenarios as the skin depth generally exceeds the height of surface roughness.

$$
C(d)=\sigma^{2} \exp \left\{-\frac{d}{\eta_{1}}\left[1-\exp \left(-\frac{d}{\eta_{2}}\right)\right]\right\} .
$$

In order to verify the effectiveness of SWM under large roughness, we use the results from HBM as the benchmark, 


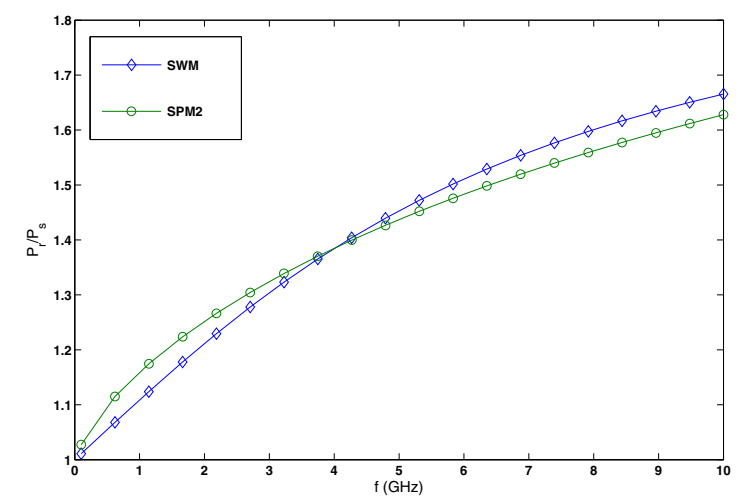

Fig. 4. SWM vs. SPM2 with extracted CF $\left(\sigma=1 \mu m, \eta_{1}=1.4 \mu m\right.$, $\left.\eta_{2}=0.53 \mu \mathrm{m}\right)$.

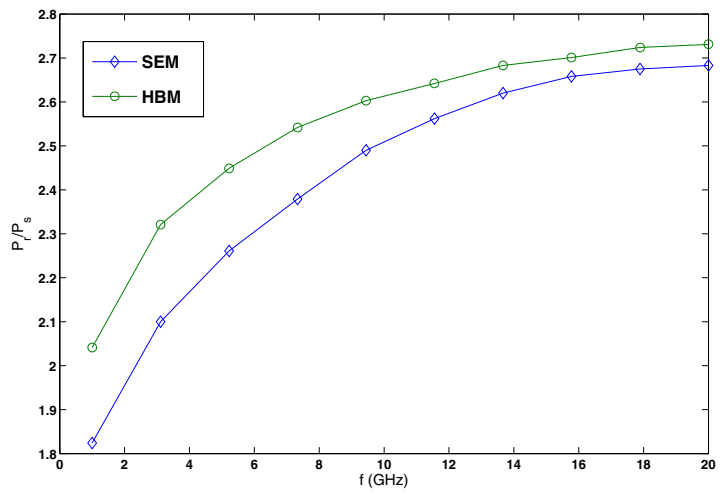

Fig. 5. SWM vs. HBM with conducting half-spheroid $\left(h_{R M S}=5.8 \mu \mathrm{m}\right.$, $d_{R M S}=9.4 \mu m, b_{R M S}=2.45 \mu m$ ).

whose accuracy has been confirmed by practical measurements for a specific setting in high frequency [5]. Since the CF of randomly distributed hemispherical bosses is not easy to extract, we only compute the loss enhancement factor of a single, deterministic conducting half-spheroid with the parameters specified in [5]. The root mean square (RMS) value of the half-spheroid base that is not explicitly given in [5] is set to be $2.45 \mu \mathrm{m}$. The testing frequency range is from $1 \mathrm{GHz}$ to $20 \mathrm{GHz}$ in order to keep the skin depth small compared to the protrusion. The discretized interval is set to be $\delta / 5$ to model the rapid variation of field inside the conductor. Simulation results are shown in Fig. 5. It can be observed that SWM matches well with HBM in very high frequency $(>15 \mathrm{GHz})$ while in an acceptable agreement with HBM in the relatively high frequencies $(1-15 \mathrm{GHz})$. The comparison proves that SWM is still valid for cases with large roughness and high frequencies. Also SPM2 completely loses it accuracy in such case as the calculated loss factor goes to a impractical value.

An important feature of 3D surface roughness modeling reported in [8] is that the loss enhancement for a 3D rough

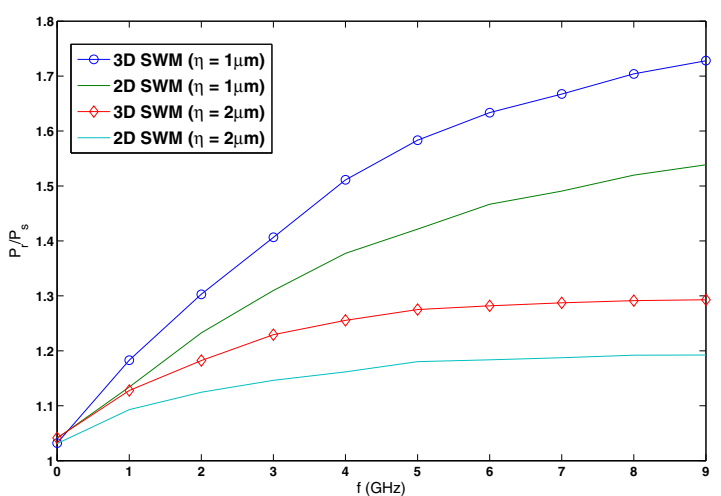

Fig. 6. 3D SWM vs. 2D SWM for Gaussian CF $(\sigma=1 \mu m, \eta=1,2 \mu m)$.

surface is much higher than a $2 \mathrm{D}$ one with the same degree of roughness. Fig. 6 compares the 3D SWM with a simplified 2D SWM formulation where the surface height is uniform along $y$ axis. The result also shows a significant increase of loss with $3 \mathrm{D}$ roughness over $2 \mathrm{D}$ roughness.

Fig. 7 shows the cumulative distribution function (CDF) of power loss factor calculated by 1st-order SSCM and 2ndSSCM. The surface is of Gaussian CF with $\sigma=\eta=1 \mu \mathrm{m}$ and $f=5 \mathrm{GHz}$. Results from the MC simulation with 5000 sampling points are used as the benchmark. It can be seen that 2nd-SSCM is sufficient to model the random nature of surface roughness. The number of sampling points of SSCM is tabulated in Table I for Gaussian CF and It shows that SSCM can offer an order of reduction in the amount sampling points when compared to the MC simulation. The slight increase in sampling points for CF (12) is due to its stronger spatial correlation.

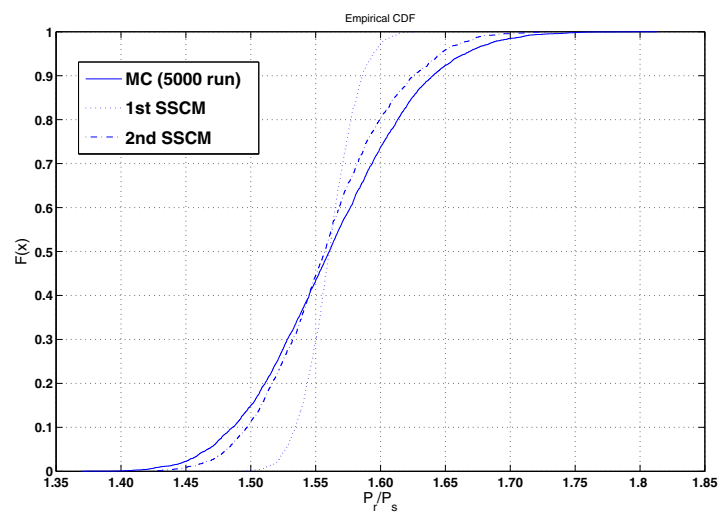

Fig. 7. CDF of $P_{r} / P_{s}(\sigma=\eta=1 \mu m, f=5 \mathrm{GHz})$.

\section{CONCLUSION}

This paper has developed a widely applicable methodology, SWM, for the simulation of rough surface loss in inter- 
TABLE I

NUMBER OF SAMPLING POINTS

\begin{tabular}{cccc}
\hline CF & MC & 1st-SSCM & 2nd-SSCM \\
\hline Gaussian & 5000 & 33 & 345 \\
\hline CF (12) & 5000 & 39 & 462
\end{tabular}

connects and packaging. The essence is to approximate the complicated EM wave scattering problem with a simplified scalar wave problem. Despite of some approximation, the SWM works surprisingly well in terms of its accuracy and applicability, which have been confirmed by comparisons with existing techniques in their respective validated regions. In particular, the proposed methodology possesses the following features:

1) It significantly expands the valid range of existing techniques like SPM2 and HBM, allowing a general simulation for different level surface roughness in a wide frequency range. It can also serve as a useful tool to test the validity of other closed-form techniques in a specific scenario.

2) Compared to the rigorous EM wave modeling, SWM leads to a remarkable computational saving by reducing the number of unknowns and using low-order basis function. In addition, SWM avoids the low-frequency problem that is difficult to handle in EM modeling and thus no additional remedies are needed.

3) The increased computational cost due to the statistical modeling of surface roughness has been well alleviated by SSCM method, which requires much less sampling points than MC simulation to achieve a satisfactory second-order accuracy.

\section{REFERENCES}

[1] H. Tanaka, "Precise measurements of dissipation factor in microwave printed circuit boards," IEEE Trans. Instrum. Meas., vol. 38, no. 2, pp. 509-514, 1989.

[2] A. Deutsch, C. W. Surovic, R. S. Krabbenhoft, and G. V. Kopcsay, "Prediction of losses caused by roughness of metallization in printedcircuit boards," IEEE Trans. Adv. Packag., vol. 30, no. 2, pp. 279-287, 2007.

[3] G. Brist, S. Hall, S. Clouser, and T. Liang, "Non-classical conductor losses due to copper foil roughness and treatment," in ECWC 10 Conf., IPC Printed Circuits Expo, 2005, pp. S19-2-1.

[4] H. Braunisch, X. Gu, A. Camacho-Bragado, and L. tsang, "Off-chip rough-metal-surface propagation loss modeling and correlation with measurements," in Proc. Intl. Conf. on Electronic Components and Technology, 2007, pp. 785-791

[5] S. Hall, S. G. Pytel, P. G. Huray, D. Hua, A. Moonshiram, G. A. Brist, and E. Sijercic, "Multigigahertz causal transmission line modeling methodology using a 3-D hemispherical surface roughness approach," IEEE Trans. Microw. Theory Tech., vol. 55, no. 12, pp. 2614-2624, 2007.

[6] S. P. Morgan, "Effect of surface roughness on Eddy current losses at microwave frequencies," J. Applied Physics, vol. 20, no. 3, pp. 352362,1949

[7] E. O. Hammerstad and F. Bekkadal, Microstrip Handbook. Trondheim, Norway: University of Trondheim, 2001.

[8] X. Gu, L. Tsang, and H. Braunisch, "Modeling effects of random rough interface on power absorption between dielectric and conductive medium in 3-D problem," IEEE Trans. Microw. Theory Tech., vol. 55, no. 3, pp. 511-517, 2007.
[9] H. Zhu, X. Zeng, W. Cai, J. Xue, and D. Zhou, "A sparse grid based spectral stochastic collocation method for variations-aware capacitance extraction of interconnects under nanometer process technology," in Proc. IEEE Intl. Conf. on Design, Automation and Test in Europe (DATE), 2007, pp. 1514-1519.

[10] K. F. Warnick and W. C. Chew, "Numerical simulation methods for rough surface scattering," J. Waves in Random Media, vol. 11, no. 1, pp. 1-30, 2001.

[11] P. Yla-Oijala and M. Taskinen, "Well-conditioned Muller formulation for electromagnetic scattering by dielectric objects," IEEE Trans. Antennas Propag., vol. 53, no. 10, pp. 3316-3323, 2005.

[12] J. S. Zhao and W. C. Chew, "Integral equation solution of Maxwell's equations from zero frequency to microwave frequencies," IEEE Trans. Antennas Propag., vol. 53, no. 10, pp. 1635-1645, 2000.

[13] H. Contopanagos, B. Dembart, M. Epton, J. J. Ottusch, V. Rokhlin, J. L. Fisher, and S. M. Wandzura, "Well-conditioned boundary integral equations for three-dimensional electromagnetic scattering," IEEE Trans. Antennas Propag., vol. 50, no. 12, pp. 1824-183, 2002.

[14] J. A. Ogilvy, Theory of Wave Scattering From Random Rough Surfaces. New York: Taylor \& Francis, 1991.

[15] L. Tsang, J. A. Kong, K. H. Ding, and C. O. Ao, Scattering of electromagnetic waves, Vol. 2, Numerical simulations. New York: Wiley, 2001.

[16] S. Oroskar, D. R. Jackson, and D. R. Wilton, "Efficient computation of the 2D periodic Greens function using the Ewald method," $J$. of Computational Physics, vol. 219, no. 15, pp. 899-911, 2006.

[17] G. Tsabarya and Y. Agnon, "Wave scattering from a rough surface: Solution by an iterative method," J. of Wave Motion, vol. 44, no. 7-8, pp. 626-648, 2007.

[18] Z. X. Li, "Wave scattering with the UV multilevel partitioning method: three-dimensional problem of dielectric rough-surface scattering," $\mathrm{Mi}$ cro and Optical Technology Letters, vol. 48, no. 7, pp. 1313-1317, 2006.

[19] S. Y. Chen, W. C. Chew, J. M. Song, and J. S. Zhao, "Analysis of low frequency scattering from penetrable scatterers," IEEE Trans. Geosci. Remote Sens., vol. 39, no. 4, pp. 726-735, 2001.

[20] S. M. Rao, D. R. Wilton, and A. W. Glisson, "Electromagnetic scattering by surfaces of arbitrary shape," IEEE Trans. Antennas Propag., vol. 30, no. 5, pp. 409-418, 1982. 